\title{
THE CATEGORY OF MV-PAIRS
}

\author{
ANTONIO DI NOLA, MICHAL HOLČAPEK, AND GEJZA JENČA
}

\begin{abstract}
An MV-pair is a pair $(B, G)$, where $B$ is a Boolean algebra and $G$ is a subgroup of the automorphism group of $B$ satisfying certain condition. Recently it was proved by one of the authors that for an MV-pair $(B, G), \sim_{G}$ is an effect-algebraic congruence and $B / \sim_{G}$ is an MV-algebra. Moreover, every MV-algebra $M$ can be represented by an MV-pair in this way.

In this paper we show that one can define a suitable category of MV-pairs in such a way that there exist a faithful functor from the category of MV-algebras to the aforementioned category and a functor in the reversed direction.
\end{abstract}

\section{INTRODUCTION}

MV-algebras stand in relation to the Eukasiewicz infinite valued logic as Boolean algebras stand in relation to classical 2-valued logic. Boolean algebras, of course, have not stayed glued to their origin in logic, their uses showing up in other areas of mathematics. Moreover, there has been extensive investigations concerning their structure.

The same can be said about MV-algebras, that is their connections to other areas of mathematics and investigations of their intrinsic structure. Classical logic, as is well known, can be analyzed in a great part by algebraic methods using the Lindenbaum algebra obtained from the formal system. For example the completeness theorem for this logic becomes equivalent to the semisimplicity of the obtained Lindenbaum algebra.

Since Chang [Ch1, Ch2], Łukasiewicz logic has also been analyzed algebraically through the associated Lindenbaum type algebra, that is the algebra of equivalence classes obtained from the relation of provable equivalence. In this case this algebra is an MV-algebra [Ch1]. Once again logical notions have an algebraic counterpart, for example, completeness relates strongly to semisimplicity [Ch1, Ch2]. However, unlike the classical case where the algebras in question are Boolean and always semisimple, not all MV-algebras are semisimple. This fact, in a sense, enriches the theory of MV-algebras.

A key relationship between Boolean algebras and MV-algebras lies on the fact that the set of all idempotent elements of an MV-algebra $M$ is a Boolean algebra, actually the greatest Boolean subalgebra of $M$. From the lattice-theoretical perspective, the Boolean algebra of idempotents is just the centre of the distributive lattice $M$. From the logical perspective, if we consider the centre as a system of

2000 Mathematics Subject Classification. Primary 06D35, secondary 03G05.

Key words and phrases. MV-algebra, Boolean algebra, MV-pair.

The paper has been partially supported by the Institutional Research Plan MŠMT 6198898701. This research is supported by grant VEGA G-1/3025/06 of MŠ SR, Slovakia and by the Slovak Research and Development Agency under the contracts No. APVT-51-032002, APVV-0071-06. 
classical propositions, the surrounding algebra $M$ can be considered as an extension of the classical logic by fuzzy (or unsharp) propositions.

This connection between MV-algebras and Boolean algebras is not the only one. Several classes of MV-algebras are described by means of Boolean algebras as it can be seen in the papers [24] [5], [23] and [14]. The present paper deals with a categorical development of the results given in [14], where a representation theorem for MV-algebras is given in terms of Boolean algebras and their automorphism group, and looking at the given MV-algebra as an MV-effect algebra. Actually, in $[14]$ is shown that given a Boolean algebra $B$ and a subgroup $G$ of its automorphism group satisfying certain conditions, the pair $(B, G)$ can be canonically associated with an MV-algebra. Such pairs are called $M V$-pairs. Furthermore, given an MValgebra $M$ and a special subgroup $G(M)$ of the automorphism group of $B(M)$, it turns out that $(B(M), G(M))$ is an MV-pair.

The notion of an MV-pair was more closely investigated by Pulmannová and Vinceková in [26]. For a given MV-pair $(B, G)$, they found a certain class of ideals of $B$ such that $(B / I, G / I)$ is an MV-pair. Independently, a similar study of certain type of $(B, G)$ pairs such that $B / G$ is an MV-algebra (called ambiguity algebras) was recently started by Vetterlein in [28].

From a purely mathematical standpoint, this development opens a new connection between two established mathematical theories.

One of them is the theory of Eukasiewicz infinite valued logic and its algebraic counterpart, MV-algebras.

The other one is the long established theory of $(B, G)$ pairs (see, for example, [19] and [27] and the references therein). For applications of $(B, G)$ pairs in recursive Boolean algebras that may be interesting for the reader, we refer to papers [21], [20], [6].

For an additional motivation, let us quote T. Vetterlein [28]:

Now, we will generalize this framework [propositional logic] so as to model statements with which a certain uncertainty is associated. Note that this is in contrast to most approaches to interpret fuzzy logics, where vagueness is the primary notion. What we propose is to model a fuzzy property by a subset of a Boolean algebra rather than a single element. Namely, we assume that we have to do with properties which are perceivable only up to the action of some group of automorphism acting on the Boolean algebra. Accordingly, a subset modelling a fuzzy property is required to be closed under the action of this group.

In this paper we show that one can define a suitable category of MV-pairs in such a way that there exist a faithful functor from the category of MV-algebras to the aforementioned category and a functor in the reversed direction.

\section{Definitions And BASiC Relationships}

An effect algebra is a partial algebra $(E ; \oplus, 0,1)$ with a binary partial operation $\oplus$ and two nullary operations 0,1 satisfying the following conditions.

(E1) If $a \oplus b$ is defined, then $b \oplus a$ is defined and $a \oplus b=b \oplus a$.

(E2) If $a \oplus b$ and $(a \oplus b) \oplus c$ are defined, then $b \oplus c$ and $a \oplus(b \oplus c)$ are defined and $(a \oplus b) \oplus c=a \oplus(b \oplus c)$.

(E3) For every $a \in E$ there is a unique $a^{\prime} \in E$ such that $a \oplus a^{\prime}=1$. 
(E4) If $a \oplus 1$ exists, then $a=0$

Effect algebras were introduced by Foulis and Bennett in their paper [8]. In their papers [15] and [16], Kôpka and Chovanec introduced an essentially equivalent structure called D-poset. Another equivalent structure, called weak orthoalgebras was introduced by Giuntini and Greuling in [9]. We refer to the monograph [7] for more information on effect algebras and similar algebraic structures.

For brevity, we denote an effect algebra $(E ; \oplus, 0,1)$ by $E$. In an effect algebra $E$, we write $a \leq b$ iff there is $c \in E$ such that $a \oplus c=b$. It is easy to check that every effect algebra is cancellative, thus $\leq$ is a partial order on $E$. In this partial order, 0 is the least and 1 is the greatest element of $E$. Moreover, it is possible to introduce a new partial operation $\ominus$; $b \ominus a$ is defined iff $a \leq b$ and then $a \oplus(b \ominus a)=b$. It can be proved that $a \oplus b$ is defined iff $a \leq b^{\prime}$ iff $b \leq a^{\prime}$. We denote the domain of $\oplus$ by $\perp$.

Let $E_{1}, E_{2}$ be effect algebras. A mapping $\phi: E_{1} \mapsto E_{2}$ is called a morphism of effect algebras iff $\phi(1)=1$ and for all $a, b \in E$, the existence of $a \oplus b$ implies the existence of $\phi(a) \oplus \phi(b)$ and $\phi(a \oplus b)=\phi(a) \oplus \phi(b)$. A morphism $\phi: E_{1} \rightarrow E_{2}$ is full iff whenever $\phi(a) \perp \phi(b)$ and $\phi(a) \oplus \phi(b) \in \phi\left(E_{1}\right)$, then there are $a_{1}, b_{1} \in E_{1}$ such that $\phi(a)=\phi\left(a_{1}\right), \phi(b)=\phi\left(b_{1}\right)$ and $a_{1} \perp b_{1}$. A morphism $\phi$ is an isomorphism iff $\phi$ is bijective and full. Note that even if both $E_{1}$ and $E_{2}$ are lattice ordered, a morphism of effect algebras need not preserve joins and meets.

An $M V$-algebra (c.f. [2], [22]) is a (2,1,0)-type algebra $(M ; \boxplus, \neg, 0)$, such that $\boxplus$ satisfies the identities $(x \boxplus y) \boxplus z=x \boxplus(y \boxplus z), x \boxplus y=y \boxplus x, x \boxplus 0=x, \neg \neg x=x$, $x \boxplus \neg 0=\neg 0$ and

$$
x \boxplus \neg(x \boxplus \neg y)=y \boxplus \neg(y \boxplus \neg x) .
$$

On every MV-algebra, a partial order $\leq$ is defined by the rule

$$
x \leq y \Longleftrightarrow y=x \boxplus \neg(x \boxplus \neg y) .
$$

In this partial order, every MV-algebra is a distributive lattice bounded by 0 and $\neg 0$.

An $M V$-effect algebra is a lattice ordered effect algebra $M$ in which, for all $a, b \in$ $M,(a \vee b) \ominus a=b \ominus(a \wedge b)$. It is proved in [4] that there is a natural, one-to one correspondence between MV-effect algebras and MV-algebras given by the following rules. Let $(M, \oplus, 0,1)$ be an MV-effect algebra. Let $\boxplus$ be a total operation given by $x \boxplus y=x \oplus\left(x^{\prime} \wedge y\right)$. Then $\left(M, \boxplus,^{\prime}, 0\right)$ is an MV-algebra. Similarly, let $(M, \boxplus, \neg, 0)$ be an MV-algebra. Restrict the operation $\boxplus$ to the pairs $(x, y)$ satisfying $x \leq y^{\prime}$ and call the new partial operation $\oplus$. Then $(M, \oplus, 0, \neg 0)$ is an MV-effect algebra.

Among lattice ordered effect algebras, MV-effect algebras can be characterized in a variety of ways. Three of them are given in the following proposition.

Proposition 1. [1], [4] Let $E$ be a lattice ordered effect algebra. The following are equivalent

(a) $E$ is an $M V$-effect algebra.

(b) For all $a, b \in E, a \wedge b=0$ implies $a \leq b^{\prime}$.

(c) For all $a, b \in E, a \ominus(a \wedge b) \leq b^{\prime}$.

(d) For all $a, b \in E$, there exist $a_{1}, b_{1}, c \in E$ such that $a_{1} \oplus b_{1} \oplus c$ exists, $a_{1} \oplus c=a$ and $b_{1} \oplus c=b$.

Notation. In what follows, we will deal with an MV-effect algebra $M$ and a Boolean algebra $B(M)$ such that $M$ is a 0,1 -sublattice of $B(M)$. In this particular 
situation, a small notational problem arises: both $M$ and $B(M)$ are MV-effect algebras, but the $\oplus, \ominus$ and ' operations on $B(M)$ and $M$ differ. To avoid confusion, we denote the partial operation of disjoint join (the $\oplus$ of Boolean algebras) on a Boolean algebra by $\dot{V}$. The partial difference of comparable elements and the complement in a Boolean algebra are denoted by $\backslash$ and ${ }^{\mathrm{C}}$, respectively.

Let $D$ be a bounded distributive lattice. Up to isomorphism, there exists a unique Boolean algebra $B(D)$ such that $D$ is a 0,1 -sublattice of $B(D)$ and $D$ generates $B(D)$ as a (Boolean) ring. This Boolean algebra is called the Boolean algebra Rgenerated by $D$. We refer to [10], section II.4, for an overview of results concerning R-generated Boolean algebras. See also [12] and [18]. For every element $x$ of $B(D)$, there exists a finite chain $x_{1} \leq \ldots \leq x_{n}$ in $D$ such that $x=x_{1}+\ldots+x_{n}$. Here, + denotes the symmetric difference, as in Boolean rings. We then say than $\left\{x_{i}\right\}_{i=1}^{n}$ is a $D$-chain representation of $x$. It is easy to see that every element of $B(D)$ has a $D$-chain representation of even length. Note that, for $n=2 k$ we have

$$
x=x_{1}+\cdots+x_{2 k}=\left(x_{2 k} \backslash x_{2 k-1}\right) \dot{\vee} \ldots \dot{\vee}\left(x_{2} \backslash x_{1}\right) .
$$

If $D_{1}, D_{2}$ are bounded distributive lattices and $\psi: D_{1} \rightarrow D_{2}$ is a 0,1-lattice homomorphism, then $\psi$ uniquely extends to a homomorphism of Boolean algebras $\psi^{*}: B\left(D_{1}\right) \rightarrow B\left(D_{2}\right)$. Similarly, if $[0, a]_{D}$ is an interval in a bounded distributive lattice $D$, then $B\left([0, a]_{D}\right)$ is naturally isomorphic to the interval $[0, a]_{B(D)}$.

Theorem 2. [13] Let $M$ be an $M V$-effect algebra. The mapping $\phi_{M}: B(M) \rightarrow M$ given by

$$
\phi_{M}(x)=\bigoplus_{i=1}^{n}\left(x_{2 i} \ominus x_{2 i-1}\right),
$$

where $\left\{x_{i}\right\}_{i=1}^{2 n}$ is a $M$-chain representation of $x$, is a surjective morphism of effect algebras.

We note that the value of $\phi_{M}(x)$ does not depend on the choice of the $M$-chain representation of $x$. Obviously, for all $x \in M,\{x, 0\}$ is a $M$-chain representation of $x$. Therefore, $\phi_{M}(x)=x \ominus 0=x$, so every $x \in M$ is a fixpoint of $\phi_{M}$.

Example 3. Let $M$ be an MV-effect algebra, which is totally ordered. By [10], Corollary II.4.19, $B(M)$ is isomorphic to the Boolean algebra of all subsets of $M$ of the form $\left[a_{1}, b_{1}\right) \dot{\cup} \ldots \dot{\cup}\left[a_{n}, b_{n}\right)$. Here, we denote $[a, b)=\{x \in M: a \leq x<b\}$. The $\phi_{M}: B(M) \rightarrow M$ morphism is then given by

$$
\phi_{M}\left(\left[a_{1}, b_{1}\right) \dot{\cup} \ldots \dot{\cup}\left[a_{n}, b_{n}\right)\right)=\left(b_{1} \ominus a_{1}\right) \oplus \ldots \oplus\left(b_{n} \ominus a_{n}\right) .
$$

Example 4. In this example, $[0,1]$ denotes the closed real unit interval. Let $C_{[0,1]}$ be the MV-effect algebra of all real continuous functions $f:[0,1] \rightarrow[0,1]$. Let $B$ be the Boolean algebra

$$
\prod_{x \in[0,1]} B([0,1])
$$

where $B([0,1])$ is the Boolean algebra generated by semiopen intervals as described in Example 3. It is obvious that $C_{[0,1]}$, as a bounded lattice, can be embedded into $B$ by a mapping $\gamma: C_{[0,1]} \rightarrow B$ given by $\gamma(f)=([0, f(x)))_{x \in[0,1]}$. The image of $C_{[0,1]}$ under $\gamma$ then generates a Boolean subalgebra of $B$, which we can identify with $B\left(C_{[0,1]}\right)$. The $\phi_{C_{[0,1]}}: B\left(C_{[0,1]}\right) \rightarrow C_{[0,1]}$ mapping can then be constructed as follows. 
Let $\left(A_{x}\right)_{x \in[0,1]} \in B\left(C_{[0,1]}\right)$. Fix $x \in[0,1]$ and write $A_{x}=\left[a_{1}, b_{1}\right) \dot{\cup} \ldots \dot{U}\left[a_{n}, b_{n}\right)$. The value of the continuous function $\phi_{C_{[0,1]}}\left(\left(A_{x}\right)_{x \in[0,1]}\right)$ at $x$ is then equal to $\left(b_{1} \ominus\right.$ $\left.a_{1}\right) \oplus \ldots \oplus\left(b_{n} \ominus a_{n}\right)$.

Let $E$ be an effect algebra. A relation $\sim$ on $E$ is a weak congruence iff the following conditions are satisfied.

(C1) $\sim$ is an equivalence relation.

(C2) If $a_{1} \sim a_{2}, b_{1} \sim b_{2}$ and $a_{1} \oplus b_{1}, a_{2} \oplus b_{2}$ exist, then $a_{1} \oplus b_{1} \sim a_{2} \oplus b_{2}$.

If $E$ is an effect algebra and $\sim$ is a weak congruence on $E$, the quotient $E / \sim$ ( $\oplus$ is defined on $E / \sim$ in an obvious way) need not to be a partial abelian monoid, since the associativity condition may fail (c.f. [11]). This fact motivates the study of sufficient conditions for a weak congruence to preserve associativity. The following condition was considered in [3].

(C5) If $a \sim b \oplus c$, then there are $b_{1}, c_{1}$ such that $b_{1} \sim b, c_{1} \sim c, b_{1} \oplus c_{1}$ exists and $a=b_{1} \oplus c_{1}$.

In [3], it was proved that for a partial abelian monoid $P$ and a weak congruence $\sim$, satisfying $(\mathrm{C} 5)$, the quotient $P / \sim$ is again a partial abelian monoid. Moreover, it is easy to prove that the eventual positivity of $P$ is preserved for such $\sim$. However, for an effect algebra $E$, the (C5) property of $\sim$ does not guarantee that the ${ }^{\prime}$ operation is preserved by $\sim$. If ' is preserved by $\sim$, that means, if condition

(C6) If $a \sim b$, then $a^{\prime} \sim b^{\prime}$.

is satisfied, then $E / \sim$ is an effect algebra. A relation on an effect algebra satisfying $(\mathrm{C} 1),(\mathrm{C} 2),(\mathrm{C} 5),(\mathrm{C} 6)$ is called an effect algebra congruence. For every effect algebra congruence $\sim$ on an effect algebra $E$, the mapping $a \rightarrow[a]_{\sim}$ is a full morphism of effect algebras.

We refer the interested reader to [25] and [11] for further details concerning congruences on effect algebras and partial abelian monoids.

\section{MV-PAIRS}

Let $B$ be a Boolean algebra. We write $\operatorname{Aut}(B)$ for the group of all automorphisms of $B$. Let $G$ be a subgroup of $\operatorname{Aut}(B)$. For $a, b \in B$, we write $a \sim_{G} b$ iff there exists $f \in G$ such that $b=f(a)$. Obviously, $\sim_{G}$ is an equivalence relation. We write $[a]_{G}$ for the equivalence class of an element $a$ of $B$.

A pair $(B, G)$, where $B$ is a Boolean algebra and $G$ is a subgroup of $\operatorname{Aut}(B)$ is called a $B G$-pair. BG-pairs are a well-established topic in the theory of Boolean algebras, see for example Chapter 15 of the handbook [17].

Let $(P, \leq)$ be a poset. Let us write,

$$
\max (P)=\{m \in P: x \geq m \Longrightarrow x=m\},
$$

that means, $\max (P)$ is the set of all maximal elements of the poset $P$.

Let $B$ be a Boolean algebra, let $G$ be a subgroup of $\operatorname{Aut}(B)$. For all $a, b \in B$, we write

$$
\begin{aligned}
& L_{G}(a, b)=\{a \wedge f(b): f \in G\} \text { and } \\
& L_{G}^{+}(a, b)=\{g(a) \wedge f(b): f, g \in G\} .
\end{aligned}
$$

Note that $L_{G}(a, b) \subseteq L_{G}^{+}(a, b)$ and that $L_{G}^{+}(a, b)$ is closed with respect to any $h \in G$; this implies that $L_{G}^{+}(a, b)$ is a union of equivalence classes of $\sim_{G}$. 
Definition 5. Let $B$ be a Boolean algebra, let $G$ be a subgroup of $\operatorname{Aut}(B)$. We say that $(B, G)$ is an $M V$-pair iff the following two conditions are satisfied.

(MVP1) For all $a, b \in B, f \in G$ such that $a \leq b$ and $f(a) \leq b$, there is $h \in G$ such that $h(a)=f(a)$ and $h(b)=b$.

(MVP2) For all $a, b \in B$ and $x \in L_{G}(a, b)$, there exists $m \in \max \left(L_{G}(a, b)\right)$ with $m \geq x$.

Example 6. For every finite Boolean algebra $B,(B, \operatorname{Aut}(B))$ is an MV-pair.

Example 7. Let $B$ be a Boolean algebra with three atoms $a_{1}, a_{2}, a_{3}$. The mapping $f$ given by

\begin{tabular}{|r||l|l|l|l|l|l|l|l|}
\hline$x$ & 0 & $a_{1}$ & $a_{2}$ & $a_{3}$ & $a_{1}^{\complement}$ & $a_{2}^{\complement}$ & $a_{3}^{\complement}$ & 1 \\
\hline$f(x)$ & 0 & $a_{2}$ & $a_{3}$ & $a_{1}$ & $a_{2}^{\complement}$ & $a_{3}^{\complement}$ & $a_{1}^{\complement}$ & 1 \\
\hline
\end{tabular}

is an automorphism of $B$ and $G=\left\{i d, f, f^{2}\right\}$ is a subgroup of $\operatorname{Aut}(B)$. However, $(B, G)$ is not an MV-pair. Indeed, we have $a_{1} \leq a_{3}^{\complement}$ and $f\left(a_{1}\right)=a_{2} \leq a_{3}^{\complement}$, but there is no $h \in G$ such that $h\left(a_{1}\right)=f\left(a_{1}\right)$ and $h\left(a_{3}^{\complement}\right)=a_{3}^{\complement}$.

Example 8. Let $B$ be the Boolean algebra of all Borel subsets of the real unit interval $[0,1]_{\mathbb{R}}$ that are unions of a finite number of intervals. (as usual, we identify the Borel sets that differ by a set of measure 0.) Let $W$ the subgroup of the permutation group of $[0,1]_{\mathbb{R}}$ that is generated by the set of all bijections $p_{a, b}$ given by

$$
p_{a, b}(x)= \begin{cases}x & \text { if } x \in[0, a], \\ a+b-x & \text { if } x \in(a, b), \\ x & \text { if } x \in[b, 1],\end{cases}
$$

where $0 \leq a \leq b \leq 1$. For every $p \in W$, let $f_{p}$ be the mapping $f_{p}: B \rightarrow B$ given by $f_{p}(X)=p(X)$ and let $G=\left\{f_{p}: p \in W\right\}$. Obviously, $G$ is a subgroup of $\operatorname{Aut}(B)$. Then $(B, G)$ is an MV-pair; the proof of this fact is a bit longer, but straightforward. Note that every $f_{p} \in G$ preserves measure.

Example 9. Let $2^{\mathbb{Z}}$ be the Boolean algebra of all subsets of $\mathbb{Z}$. Then $\left(2^{\mathbb{Z}}, \operatorname{Aut}\left(2^{\mathbb{Z}}\right)\right)$ is not an MV-pair. Indeed, let $f \in \operatorname{Aut}\left(2^{\mathbb{Z}}\right)$ be the automorphism of $2^{\mathbb{Z}}$ associated with the permutation $f(n)=n+1$. Let $A=B=\mathbb{N}$. We see that $f(A)=A \backslash\{0\}$, $A \subseteq B$ and $f(A) \subseteq B$. However, there is no $h \in \operatorname{Aut}\left(2^{\mathbb{Z}}\right)$ such that $h(A)=f(A)$ and $h(B)=B$, simply because $A=B$ implies that $h(A)=h(B)$, but $f(A) \neq B$.

Let us summarize the main results from the paper [14] we shall need.

Theorem 10. Let $(B, G)$ be an $M V$-pair. Then

(a) $\sim_{G}$ is an effect algebra congruence,

(b) $B / \sim_{G}$ is an $M V$-effect algebra,

(c) for all $a, b \in B$,

$$
[a]_{G} \wedge[b]_{G}=\max \left(L_{G}^{+}(a, b)\right)
$$

where the $=i s$ a set equality,

(d) $\max \left(L_{G}(a, b)\right) \subseteq \max \left(L_{G}^{+}(a, b)\right)$.

For an MV-algebra $M$, we write

$$
G(M)=\left\{f \in \operatorname{Aut}(B(M)): \text { for all } x \in B(M), \phi_{M}(x)=\phi_{M}(f(x))\right\} .
$$

Theorem 11. Let $M$ be an $M V$-algebra. 
(a) $(B(M), G(M))$ is an $M V$-pair.

(b) For all $x, y \in B(M), x \sim_{G(M)}$ y iff $\phi_{M}(x)=\phi_{M}(y)$.

(c) $B(M) / G(M)$ is isomorphic to $M$, where the isomorphism is given by

$$
\beta_{M}\left([x]_{G(M)}\right)=\phi_{M}(x) .
$$

\section{Premorphisms of MV-PAirs}

Definition 12. Let $\left(B_{1}, G_{1}\right),\left(B_{2}, G_{2}\right)$ be MV-pairs. A mapping $\psi_{B}: B_{1} \rightarrow B_{2}$ is called a premorphism of $M V$-pairs iff the following conditions are satisfied.

(1) $\psi_{B}$ is a morphism of Boolean algebras.

(2) For every $f_{1} \in G_{1}$ and $x \in B_{1}, \psi_{B}(x)=0$ implies that $\psi_{B}\left(f_{1}(x)\right)=0$.

Proposition 13. Let $\left(B_{1}, G_{1}\right),\left(B_{2}, G_{2}\right)$ be $M V$-pairs and let $\psi_{B}: B_{1} \rightarrow B_{2}$ be a premorphism of $M V$-pairs. Then for every $f_{1} \in G_{1}$, the mapping $\psi_{G}\left(f_{1}\right)$ : $\psi_{B}\left(B_{1}\right) \rightarrow \psi_{B}\left(B_{1}\right)$ given by

$$
\psi_{G}\left(f_{1}\right)\left(\psi_{B}(x)\right)=\psi_{B}\left(f_{1}(x)\right)
$$

is an automorphism of the Boolean algebra $\psi_{B}\left(B_{1}\right)$.

Proof. Let us first prove that $\psi_{G}\left(f_{1}\right)$ is well-defined, that means, that the value of $\psi_{G}(f)$ at an element $\psi_{B}(x)$ of the set $\psi_{B}\left(B_{1}\right)$ does not depend on the choice of $x$.

Let $x, y \in B_{1}$ be such that $\psi_{B}(x)=\psi_{B}(y)$. Since $\psi_{B}(x)+\psi_{B}(y)=0, \psi_{B}(x+y)=$ 0 . By condition $(2), \psi_{B}\left(f_{1}(x+y)\right)=0$ and we see that

$$
\psi_{B}\left(f_{1}(x+y)\right)=\psi_{B}\left(f_{1}(x)+f_{1}(y)\right)=\psi_{B}\left(f_{1}(x)\right)+\psi_{B}\left(f_{1}(y)\right)=0 .
$$

Therefore, $\psi_{B}\left(f_{1}(x)\right)=\psi_{B}\left(f_{1}(y)\right)$ and the value of $\psi_{G}\left(f_{1}\right)$ is well defined.

Let us prove that every $\psi_{G}\left(f_{1}\right)$ is injective. Let $\psi_{B}(x), \psi_{B}(y) \in \psi_{B}\left(B_{1}\right)$ be such that $\psi_{G}\left(f_{1}\right)\left(\psi_{B}(x)\right)=\psi_{G}\left(f_{1}\right)\left(\psi_{B}(y)\right)$, that means, $\psi_{B}\left(f_{1}(x)\right)=\psi_{B}\left(f_{1}(y)\right)$. Similarly as in the previous part of the proof, this implies that $\psi_{B}\left(f_{1}(x+y)\right)=0$. Therefore, by condition (2),

$$
\psi_{B}\left(f_{1}^{-1}\left(f_{1}(x+y)\right)\right)=\psi_{B}(x+y)=\psi_{B}(x)+\psi_{B}(y)=0
$$

and we see that $\psi_{B}(x)=\psi_{B}(y)$.

Let us prove that every $\psi_{G}\left(f_{1}\right)$ is surjective. Let $u \in \psi_{B}\left(B_{1}\right)$. Then $u=\psi_{B}(x)$ for some $x \in B_{1}$ and we see that

$$
\psi_{G}\left(f_{1}\right)\left(f_{1}^{-1}(x)\right)=\psi_{B}\left(f_{1}\left(f_{1}^{-1}(x)\right)\right)=\psi_{B}(x)=u .
$$

It remains to prove that every $\psi_{G}\left(f_{1}\right)$ is an endomorphism of the Boolean algebra $\psi_{B}\left(B_{1}\right)$. This requires only a simple diagram chasing, let us prove (for example) that for every $f_{1} \in G_{1}, \psi_{G}\left(f_{1}\right)$ is a $\vee$-endomorphism. Let $x, y \in B_{1}$. Then

$$
\begin{aligned}
& \psi_{G}\left(f_{1}\right)\left(\psi_{B}(x) \vee \psi_{B}(y)\right)=\psi_{G}\left(f_{1}\right)\left(\psi_{B}(x \vee y)\right)=\psi_{B}\left(f_{1}(x \vee y)\right)= \\
= & \psi_{B}\left(f_{1}(x)\right) \vee \psi_{B}\left(f_{1}(y)\right)=\psi_{G}(f)\left(\psi_{B}(x)\right) \vee \psi_{G}(f)\left(\psi_{B}(y)\right)
\end{aligned}
$$

The proof that $\psi_{G}\left(f_{1}\right)$ preserves all the other Boolean operations is very similar.

Proposition 14. Let $\left(B_{1}, G_{1}\right),\left(B_{2}, G_{2}\right)$ be $M V$-pairs and let $\psi_{B}: B_{1} \rightarrow B_{2}$ be a premorphism of $M V$-pairs. Then $\psi_{G}: G_{1} \rightarrow \operatorname{Aut}\left(\psi_{B}\left(B_{1}\right)\right)$ is a group morphism and

$$
\operatorname{Ker}\left(\psi_{G}\right)=\left\{f_{1} \in G_{1}: f_{1}(x)+x \in \operatorname{Ker}\left(\psi_{B}\right) \text {, for all } x \in B_{1}\right\} \text {. }
$$


Proof. Let $f_{1}, g_{1} \in G_{1}$. We need to prove that $\psi_{G}\left(f_{1} \circ g_{1}\right)=\psi_{G}\left(f_{1}\right) \circ \psi_{G}\left(g_{1}\right)$.

For all $x \in B_{1}$,

$$
\begin{aligned}
& \psi_{G}\left(f_{1} \circ g_{1}\right)\left(\psi_{B}(x)\right)=\psi_{B}\left(\left(f_{1} \circ g_{1}\right)(x)\right)=\psi_{B}\left(f_{1}\left(g_{1}(x)\right)\right)= \\
= & \psi_{G}\left(f_{1}\right)\left(\psi_{B}\left(g_{1}(x)\right)\right)=\psi_{G}\left(f_{1}\right)\left(\psi_{G}\left(g_{1}\right)\left(\psi_{B}(x)\right)\right)= \\
= & \left(\psi_{G}\left(f_{1}\right) \circ \psi_{G}\left(g_{1}\right)\right)\left(\psi_{B}(x)\right),
\end{aligned}
$$

hence $\psi_{G}$ is a group morphism.

Let $f_{1} \in \operatorname{Ker}\left(\psi_{G}\right)$, that means, $\psi_{G}\left(f_{1}\right)=\mathrm{id}_{\psi_{B}\left(B_{1}\right)}$. For all $x \in B_{1}, \psi_{G}\left(f_{1}\right)\left(\psi_{B}(x)\right)=$ $\psi_{B}(x)$. This is equivalent to $\psi_{B}\left(f_{1}(x)\right)=\psi_{B}(x)$ and, since $\psi_{B}$ is a morphism of Boolean algebras, $\psi_{B}\left(f_{1}(x)+x\right)=0$.

Let $f_{1} \in G_{1}$ be such that, for all $x \in B_{1}, f_{1}(x)+x \in \operatorname{Ker}\left(\psi_{B}\right)$. We need to prove that $f_{1} \in \operatorname{Ker}\left(\psi_{G}\right)$, that is, $\psi_{G}\left(f_{1}\right)=\operatorname{id}_{\psi_{B}\left(B_{1}\right)}$. Let $u \in \psi_{B}\left(B_{1}\right)$. There is $x \in B_{1}$ such that $\psi_{B}(x)=u$ and

$$
\psi_{G}\left(f_{1}\right)(u)=\psi_{G}\left(f_{1}\right)\left(\psi_{B}(x)\right)=\psi_{B}\left(f_{1}(x)\right) .
$$

As $\psi_{B}\left(f_{1}(x)\right)+\psi_{B}(x)=\psi_{B}\left(f_{1}(x)+x\right)=0, \psi_{B}\left(f_{1}(x)\right)=\psi_{B}(x)=u$. Therefore, $\psi_{G}\left(f_{1}\right)(u)=u$ and we see that $\psi_{G}\left(f_{1}\right)=\operatorname{id}_{\psi_{B}\left(B_{1}\right)}$.

\section{WEAK MORPHISMS OF MV-PAIRS}

Since we want to develop a proper notion of morphism of MV-pairs, we need to strengthen the notion of premorphism in order to be able to associate a map $\Delta\left(\psi_{B}\right): B_{1} / G_{1} \rightarrow B_{2} / G_{2}$ with $\psi_{B}$. The condition is defined in a natural way, as follows.

Definition 15. Let $\left(B_{1}, G_{1}\right),\left(B_{2}, G_{2}\right)$ be MV-pairs. A mapping $\psi_{B}: B_{1} \rightarrow B_{2}$ is called a weak morphism of MV-pairs iff the following conditions are satisfied.

(1) $\psi_{B}$ is a morphism of Boolean algebras.

(3) For all $x, y \in B_{1}, x \sim_{G_{1}} y$ implies that $\psi_{B}(x) \sim_{G_{2}} \psi_{B}(y)$.

If $\psi_{B}$ is a weak morphism, then $\Delta\left(\psi_{B}\right): B_{1} / G_{1} \rightarrow B_{2} / G_{2}$ is given by

$$
\Delta\left(\psi_{B}\right)\left([x]_{G_{1}}\right)=\left[\psi_{B}(x)\right]_{G_{2}} .
$$

Obviously, condition (3) ensures that the mapping $\Delta\left(\psi_{B}\right)$ is well defined.

Proposition 16. Every weak morphism of $M V$-pairs is a premorphism of $M V$ pairs.

Proof. We need to prove condition (2) of Definition 12. Let $\psi_{B}$ be a weak morphism of MV-pairs $\left(B_{1}, G_{1}\right),\left(B_{2}, G_{2}\right)$. Let $f_{1} \in G_{1}, x \in B_{1}$ and suppose that $\psi_{B}(x)=$ 0 . Put $y=f_{1}(x)$, we have $x \sim_{G_{1}} y$ and hence, by condition $(3), \psi_{B}(x) \sim_{G_{2}}$ $\psi_{B}\left(f_{1}(x)\right)$. Since $\psi_{B}(x)=0,0 \sim_{G_{2}} \psi_{B}\left(f_{1}(x)\right)$. Thus, there is a $f_{2} \in G_{2}$ such that $f_{2}\left(\psi_{B}\left(f_{1}(x)\right)\right)=0$. Since $f_{2}$ is an automorphism of $B_{2}, \psi_{B}\left(f_{1}(x)\right)=0$.

Proposition 17. Let $\left(B_{1}, G_{1}\right),\left(B_{2}, G_{2}\right)$ be $M V$-pairs and let $\psi_{B}: B_{1} \rightarrow B_{2}$ be a weak morphism of $M V$-pairs. Then $\Delta\left(\psi_{B}\right)$ is a morphism of effect algebras.

Proof. Let $x, y \in B_{1}$ be such that $[x]_{G_{1}} \perp[y]_{G_{1}}$. Without loss of generality, we may assume that $x \perp y$ in $B_{1}$ and we may compute

$$
\begin{aligned}
& \Delta\left(\psi_{B}\right)\left([x]_{G_{1}} \oplus[y]_{G_{1}}\right)=\Delta\left(\psi_{B}\right)\left([x \oplus y]_{G_{1}}\right)=\left[\psi_{B}(x \oplus y)\right]_{G_{2}}= \\
= & {\left[\psi_{B}(x) \oplus \psi_{B}(y)\right]_{G_{2}}=\left[\psi_{B}(x)\right]_{G_{2}} \oplus\left[\psi_{B}(y)\right]_{G_{2}}=\Delta\left(\psi_{B}\right)\left([x]_{G_{1}}\right) \oplus \Delta\left(\psi_{B}\right)\left([y]_{G_{1}}\right) . }
\end{aligned}
$$

It is easy to see that $\Delta\left(\psi_{B}\right)$ preserves the unit. 
Let $\psi: M_{1} \rightarrow M_{2}$ be a morphism of MV-algebras. Since $\psi$ is a bounded lattice morphism, it uniquely extends to a morphism of Boolean algebras $\nabla(\psi): B\left(M_{1}\right) \rightarrow$ $B\left(M_{2}\right)$.

The following lemma is a generalization of Lemma 18 from [26], where only the case of surjective $\psi$ is considered. However, it is easy to observe that this restriction is not necessary, so the proof here is essentially the same as in [26].

Lemma 18. For every morphism $\psi: M_{1} \rightarrow M_{2}$ of $M V$-algebras, the diagram

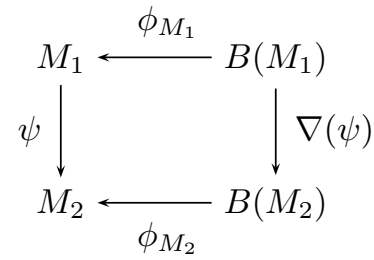

commutes.

Proof. Let $x \in B\left(M_{1}\right)$, let $\left(x_{i}\right)_{i=1}^{2 n}$ be its $M_{1}$-chain representation:

$$
x=\left(x_{2} \backslash x_{1}\right) \dot{\vee} \ldots \dot{\vee}\left(x_{2 n} \backslash x_{2 n-1}\right) .
$$

Then, by the definition of $\phi_{M_{1}}$,

$$
\begin{aligned}
& \phi_{M_{1}}(x)=\phi_{M_{1}}\left(\left(x_{2} \backslash x_{1}\right) \dot{\vee} \ldots \dot{\vee}\left(x_{2 n} \backslash x_{2 n-1}\right)\right)= \\
= & \left(x_{2} \ominus x_{1}\right) \oplus \cdots \oplus\left(x_{2 n} \ominus x_{2 n-1}\right)=\bigoplus_{i=1}^{n}\left(x_{2 i} \ominus x_{2 i-1}\right)
\end{aligned}
$$

where the latter term is in $M_{1}$.

Since $\psi$ is a morphism of MV-algebras,

$$
\psi\left(\phi_{M_{1}}(x)\right)=\psi\left(\bigoplus_{i=1}^{n}\left(x_{2 i} \ominus x_{2 i-1}\right)\right)=\bigoplus_{i=1}^{n}\left(\psi\left(x_{2 i}\right) \ominus \psi\left(x_{2 i-1}\right)\right) .
$$

On the other hand, since $\nabla(\psi)$ is a morphism of Boolean algebras,

$$
\nabla(\psi)(x)=\left(\nabla(\psi)\left(x_{2}\right) \backslash \nabla(\psi)\left(x_{1}\right)\right) \dot{\vee} \ldots \dot{\vee}\left(\nabla(\psi)\left(x_{2 n}\right) \backslash \nabla(\psi)\left(x_{2 n-1}\right)\right) .
$$

Since $\nabla(\psi)$ is an extension of the mapping $\psi$, for all $y \in M_{1}$ we have $\nabla(\psi)(y)=\psi(y)$ and hence

$$
\nabla(\psi)(x)=\left(\psi\left(x_{2}\right) \backslash \psi\left(x_{1}\right)\right) \dot{V} \ldots \dot{V}\left(\psi\left(x_{2 n}\right) \backslash \psi\left(x_{2 n-1}\right)\right) .
$$

Finally, by the definition of $\phi_{M_{2}}$

$$
\phi_{M_{2}}(\nabla(\psi)(x))=\bigoplus_{i=1}^{n}\left(\psi\left(x_{2 i}\right) \ominus \psi\left(x_{2 i-1}\right)\right) .
$$

Proposition 19. For every morphism $\psi: M_{1} \rightarrow M_{2}$ of $M V$-algebras, $\nabla(\psi)$ : $B\left(M_{1}\right) \rightarrow B\left(M_{2}\right)$ is a weak morphism of $M V$-pairs $\left(B\left(M_{1}\right), G\left(M_{1}\right)\right),\left(B\left(M_{2}\right), G\left(M_{2}\right)\right)$.

Proof. The condition (1) of Definition 15 is automatically satisfied.

To prove condition (3) of Definition 15, let $x, y \in B_{1}$ be such that $x \sim_{G\left(M_{1}\right)} y$. By the definition of $G\left(M_{1}\right)$, this is equivalent with $\phi_{M_{1}}(x)=\phi_{M_{1}}(y)$. We need to prove that $\nabla(\psi)(x) \sim_{G_{2}} \nabla(\psi)(y)$, that means,

$$
\phi_{M_{2}}(\nabla(\psi)(x))=\phi_{M_{2}}(\nabla(\psi)(y)) .
$$


Clearly, this follows from $\phi_{M_{1}}(x)=\phi_{M_{1}}(y)$ by Lemma 18:

$$
\phi_{M_{2}}(\nabla(\psi)(x))=\psi\left(\phi_{M_{1}}(x)\right)=\psi\left(\phi_{M_{2}}(x)\right)=\phi_{M_{2}}(\nabla(\psi)(y)) \text {. }
$$

There exists a premorphism of MV-pairs that is not a weak morphism, as the following example shows.

Example 20. Consider the MV-pairs $\left(2^{2}\right.$, Aut $\left.\left(2^{2}\right)\right)$ and $\left(2^{3}\right.$, Aut $\left.\left(2^{3}\right)\right)$. Let $\psi_{B}$ be any injective morphism of Boolean algebras $\psi_{B}: 2^{2} \rightarrow 2^{3}$. Since $\operatorname{Ker}\left(\psi_{B}\right)=\{0\}$, $\psi_{B}$ is a premorphism of MV-pairs.

Suppose that $\psi_{B}$ is a weak morphism. By Proposition 19, $\Delta\left(\psi_{B}\right): 2^{2} / \operatorname{Aut}\left(2^{2}\right) \rightarrow$ $2^{3} / \operatorname{Aut}\left(2^{3}\right)$ is then a morphism of effect algebras. However, $2^{2} / \operatorname{Aut}\left(2^{2}\right)$ is a 3 element chain and $2^{3} / \operatorname{Aut}\left(2^{3}\right)$ is a 4 -element chain, and it is easy to check that there is no such morphism of effect algebras.

\section{Morphisms OF MV-PAIRS}

In this section, we will define a notion of morphism of MV-pairs. A morphism of MV-pairs is a premorphism of MV-pairs satisfying an additional condition; we need this condition to prove that $\Delta\left(\psi_{B}\right)$ is a morphism of MV-algebras. We also prove that for every morphism $\psi$ of MV-algebras, $\nabla(\psi)$ is a morphism of MV-pairs.

Definition 21. Let $\psi_{B}$ be a weak morphism of MV-pairs $\left(B_{1}, G_{1}\right),\left(B_{2}, G_{2}\right)$. We say that $\psi_{B}$ is a morphism of $M V$-pairs iff

(4) For all $x, y \in B_{1}$ and $f_{2} \in G_{2}$ there exists $f_{1} \in G_{1}$ such that

$$
\left[\psi_{B}(x) \wedge f_{2}\left(\psi_{B}(y)\right)\right]_{G_{2}} \leq\left[\psi_{B}\left(x \wedge f_{1}(y)\right)\right]_{G_{2}}
$$

Lemma 22. Let $M_{1}, M_{2}$ be $M V$-algebras, let $\alpha: M_{1} \rightarrow M_{2}$ be a morphism of effect algebras. Suppose that, for all $x, y \in M_{1}, \alpha(u) \wedge \alpha(v) \leq \alpha(u \wedge v)$. Then $\alpha$ is a morphism of $M V$-algebras.

Proof. It suffices to prove that $\alpha$ preserves $\wedge$. Since $\alpha$ is a morphism of effect algebras, $\alpha$ is isotone. Therefore, $\alpha(u) \wedge \alpha(v) \geq \alpha(u \wedge v)$ and $\alpha(u) \wedge \alpha(v)=$ $\alpha(u \wedge v)$.

Theorem 23. Let $\psi_{B}$ be a morphism of $M V$-pairs $\left(B_{1}, G_{1}\right),\left(B_{2}, G_{2}\right)$. Then $\Delta\left(\psi_{B}\right)$ is a morphism of $M V$-algebras.

Proof. By Proposition 17, $\Delta\left(\psi_{B}\right)$ is a morphism of effect algebras. By Lemma 22, it suffices to prove that for all $x, y \in B_{1}$,

$$
\Delta\left(\psi_{B}\right)\left([x]_{G_{1}}\right) \wedge \Delta\left(\psi_{B}\right)\left([y]_{G_{1}}\right) \leq \Delta\left(\psi_{B}\right)\left([x]_{G_{1}} \wedge[y]_{G_{1}}\right) .
$$

By definition of $\Delta\left(\psi_{B}\right)$,

$$
\Delta\left(\psi_{B}\right)\left([x]_{G_{1}}\right) \wedge \Delta\left(\psi_{B}\right)\left([y]_{G_{1}}\right)=\left[\psi_{B}(x)\right]_{G_{2}} \wedge\left[\psi_{B}(y)\right]_{G_{2}} .
$$

By Theorem 10 (c) and (d), there is $m \in \max \left(L_{G_{2}}\left(\psi_{B}(x), \psi_{B}(y)\right)\right)$ such that

$$
\left[\psi_{B}(x)\right]_{G_{2}} \wedge\left[\psi_{B}(y)\right]_{G_{2}}=[m]_{G_{2}} .
$$

As $m \in L_{G_{2}}\left(\psi_{B}(x), \psi_{B}(y)\right)$, there is $f_{2} \in G_{2}$ such that

$$
m=\psi_{B}(x) \wedge f_{2}\left(\psi_{B}(y)\right) .
$$

By condition (4), there is $f_{1} \in G_{1}$ such that

$$
[m]_{G_{2}} \leq\left[\psi_{B}\left(x \wedge f_{1}(y)\right)\right]_{G_{2}}
$$


By definition of $\Delta\left(\psi_{B}\right)$,

$$
\left[\psi_{B}\left(x \wedge f_{1}(y)\right)\right]_{G_{2}}=\Delta\left(\psi_{B}\right)\left(\left[x \wedge f_{1}(y)\right]_{G_{1}}\right) .
$$

Since $[x]_{G_{1}},[y]_{G_{1}} \geq\left[x \wedge f_{1}(y)\right]_{G_{1}}$,

$$
[x]_{G_{1}} \wedge[y]_{G_{1}} \geq\left[x \wedge f_{1}(y)\right]_{G_{1}} .
$$

Since $\Delta\left(\psi_{B}\right)$ is isotone,

$$
\Delta\left(\psi_{B}\right)\left([x]_{G_{1}} \wedge[y]_{G_{1}}\right) \geq \Delta\left(\psi_{B}\right)\left(\left[x \wedge f_{1}(y)\right]_{G_{1}}\right) .
$$

Moreover, as

$$
\Delta\left(\psi_{B}\right)\left(\left[x \wedge f_{1}(y)\right]_{G_{1}}\right) \geq[m]_{G_{2}}
$$

and

$$
[m]_{G_{2}}=\left[\psi_{B}(x)\right]_{G_{2}} \wedge\left[\psi_{B}(y)\right]_{G_{2}}=\Delta\left(\psi_{B}\right)\left([x]_{G_{1}}\right) \wedge \Delta\left(\psi_{B}\right)\left([y]_{G_{1}}\right),
$$

we see that

$$
\Delta\left(\psi_{B}\right)\left([x]_{G_{1}} \wedge[y]_{G_{1}}\right) \geq \Delta\left(\psi_{B}\right)\left([x]_{G_{1}}\right) \wedge \Delta\left(\psi_{B}\right)\left([y]_{G_{1}}\right) .
$$

By Lemma 22 and Theorem 23, this is implies that $\Delta\left(\psi_{B}\right)$ is a morphism of MValgebras.

We need to prove that for a morphism $\psi$ of MV-algebras, $\nabla(\psi)$ is a morphism of MV-pairs. The proof is divided to a sequence of lemmas that will be useful later.

Let $\psi: M_{1} \rightarrow M_{2}$ be a morphism of effect algebras. Let us define a mapping $\widehat{\psi}: B\left(M_{1}\right) / G\left(M_{1}\right) \rightarrow B\left(M_{2}\right) / G\left(M_{2}\right)$ by

$$
\widehat{\psi}\left([a]_{G\left(M_{1}\right)}\right)=\left[\psi\left(\phi_{M_{1}}(a)\right)\right]_{G\left(M_{2}\right)}
$$

Lemma 24. Let $\psi: M_{1} \rightarrow M_{2}$ be a morphism of $M V$-algebras. Consider (see Theorem 11) the pair of isomorphisms of $M V$-algebras $\beta_{M_{1}}, \beta_{M_{2}}$, where each $\beta_{M_{i}}$ : $B\left(M_{i}\right) / G\left(M_{i}\right) \rightarrow M_{i}$ is given by

$$
\beta_{M_{i}}\left([a]_{G\left(M_{i}\right)}\right)=\phi_{M_{i}}(a) .
$$

The diagram

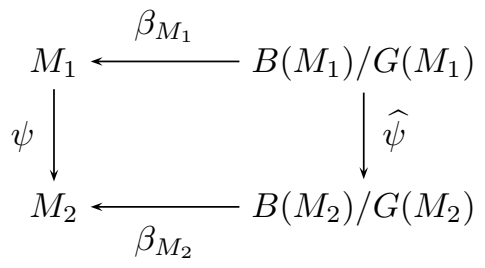

commutes.

Proof. We see that

$$
\psi\left(\beta_{M_{1}}\left([a]_{G\left(M_{1}\right)}\right)\right)=\psi\left(\phi_{M_{1}}(a)\right)
$$

and that

$$
\beta_{M_{2}}\left(\widehat{\psi}\left([a]_{G\left(M_{1}\right)}\right)\right)=\beta_{M_{2}}\left(\left[\psi\left(\phi_{M_{1}}(a)\right)\right]_{G\left(M_{2}\right)}\right)=\phi_{M_{2}}\left(\psi\left(\phi_{M_{1}}(a)\right)\right) .
$$

However, $\psi\left(\phi_{M_{1}}(a)\right) \in M_{2}$ and $\phi_{M_{2}}$ is the identity on $M_{2}$, so

$$
\phi_{M_{2}}\left(\psi\left(\phi_{M_{1}}(a)\right)\right)=\psi\left(\phi_{M_{1}}(a)\right) \text {. }
$$

Corollary 25. $\widehat{\psi}$ is a morphism of $M V$-algebras. 
Proof. $\widehat{\psi}=\beta_{M_{2}}^{-1} \circ \psi \circ \beta_{M_{1}}$.

Lemma 26. Let $\psi: M_{1} \rightarrow M_{2}$ be a morphism of $M V$-algebras. For all $a \in B\left(M_{1}\right)$,

$$
[\nabla(\psi)(a)]_{G\left(M_{2}\right)}=\widehat{\psi}\left([a]_{G\left(M_{1}\right)}\right) .
$$

Proof. We need to prove

$$
[\nabla(\psi)(a)]_{G\left(M_{2}\right)}=\left[\psi\left(\phi_{M_{1}}(a)\right)\right]_{G\left(M_{2}\right)} .
$$

By definition of $G\left(M_{2}\right)$, this is equivalent to

$$
\phi_{M_{2}}(\nabla(\psi)(a))=\phi_{M_{2}}\left(\psi\left(\phi_{M_{1}}(a)\right)\right) .
$$

Since $\phi_{M_{2}}$ is the identity on $M_{2}$,

$$
\phi_{M_{2}}\left(\psi\left(\phi_{M_{1}}(a)\right)\right)=\psi\left(\phi_{M_{1}}(a)\right) .
$$

It remains to prove

$$
\phi_{M_{2}}(\nabla(\psi)(a))=\psi\left(\phi_{M_{1}}(a)\right),
$$

but this is clear by Lemma 18 .

Corollary 27. For every morphism of $M V$-algebras $\psi: M_{1} \rightarrow M_{2}, \widehat{\psi}=\Delta(\nabla(\psi))$.

Proof. Let $[a] \in B\left(M_{1}\right) / G\left(M_{1}\right)$. By definition of $\Delta$,

$$
\Delta(\nabla(\psi))\left([a]_{G\left(M_{1}\right)}\right)=[\nabla(\psi)(a)]_{G\left(M_{2}\right)} .
$$

By Lemma 26,

$$
[\nabla(\psi)(a)]_{G\left(M_{2}\right)}=\widehat{\psi}\left([a]_{G\left(M_{1}\right)}\right)
$$

Theorem 28. For every morphism of $M V$-algebras $\psi: M_{1} \rightarrow M_{2}, \nabla(\psi)$ is a morphism of $M V$-pairs $\left(B\left(M_{1}\right), G\left(M_{1}\right)\right),\left(B\left(M_{2}\right), G\left(M_{2}\right)\right)$.

Proof. We have already proved (Proposition 19$)$ that $\nabla(\psi)$ is a weak morphism. It remains to prove the condition (4) of Definition 21.

Let $x, y \in B\left(M_{1}\right)$, let $f_{2} \in G\left(M_{2}\right)$. Take $x \wedge f_{1}(y) \in \max (L(x, y))$. By Theorem 10 ,

$$
\left[x \wedge f_{1}(y)\right]_{G\left(M_{1}\right)}=[x]_{G\left(M_{1}\right)} \wedge[y]_{G\left(M_{1}\right)} .
$$

Applying Lemma 26 and Corollary 25, we obtain

$$
\begin{aligned}
{\left[\nabla(\psi)\left(x \wedge f_{1}(y)\right)\right]_{G\left(M_{2}\right)} } & =\widehat{\psi}\left(\left[x \wedge f_{1}(y)\right]_{G\left(M_{1}\right)}\right)=\widehat{\psi}\left([x]_{G\left(M_{1}\right)} \wedge[y]_{G\left(M_{1}\right)}\right)= \\
\widehat{\psi}\left([x]_{G\left(M_{1}\right)}\right) & \wedge \widehat{\psi}\left([y]_{G\left(M_{1}\right)}\right)=[\nabla(\psi)(x)]_{G\left(M_{2}\right)} \wedge[\nabla(\psi)(y)]_{G\left(M_{2}\right)}
\end{aligned}
$$

Obviously,

$$
[\nabla(\psi)(x)]_{G\left(M_{2}\right)} \geq\left[\nabla(\psi)(x) \wedge f_{2}(\nabla(\psi)(y))\right]_{G\left(M_{2}\right)}
$$

and

$$
[\nabla(\psi)(y)]_{G\left(M_{2}\right)}=\left[f_{2}(\nabla(\psi)(y))\right]_{G\left(M_{2}\right)} \geq\left[\nabla(\psi)(x) \wedge f_{2}(\nabla(\psi)(y))\right]_{G\left(M_{2}\right)} .
$$

Therefore,

$$
[\nabla(\psi)(x)]_{G\left(M_{2}\right)} \wedge[\nabla(\psi)(y)]_{G\left(M_{2}\right)} \geq\left[\nabla(\psi)(x) \wedge f_{2}(\nabla(\psi)(y))\right]_{G\left(M_{2}\right)} .
$$




\section{Categorical topics}

In this section, we prove that the class of MV-pairs equipped with morphisms of MV-pairs in the sense of 21 forms a category and that $\Delta$ and $\nabla$ are functors.

Lemma 29. Let $\psi_{B}$ be a morphism of $M V$-pairs $\left(B_{1}, G_{1}\right) \rightarrow\left(B_{2}, G_{2}\right)$. Let $y \in B_{1}$, $f_{2} \in G_{2}$. There exists $f_{1} \in G_{1}$ such that

$$
\left[f_{2}\left(\psi_{B}(y)\right)\right]_{G_{2}} \leq\left[\psi_{B}\left(f_{1}(y)\right)\right]_{G_{2}}
$$

Proof. Put $x=1$ in condition (4).

Proposition 30. The class of $M V$-pairs, equipped with morphisms of $M V$-pairs, forms a category.

Proof. We need to prove that a composition of morphisms of MV-pairs is a morphism of MV-pairs.

Let

$$
\begin{aligned}
& \psi_{B}^{1}:\left(B_{1}, G_{1}\right) \rightarrow\left(B_{2}, G_{2}\right) \\
& \psi_{B}^{2}:\left(B_{2}, G_{2}\right) \rightarrow\left(B_{3}, G_{3}\right)
\end{aligned}
$$

be morphisms of MV-pairs. Clearly, $\psi_{B}^{2} \circ \psi_{B}^{1}$ satisfies the condition (1).

For the proof of condition (3), let $x, y \in B_{1}$. We need to prove that $x \sim_{G_{1}} y$ implies that

$$
\psi_{B}^{2}\left(\psi_{B}^{1}(x)\right) \sim_{G_{3}} \psi_{B}^{2}\left(\psi_{B}^{1}(y)\right) .
$$

This follows easily from the fact that $\psi_{B}^{1}, \psi_{B}^{2}$ satisfy the condition (3).

To prove condition (4), let $x, y \in B_{1}$ and let $f_{3} \in G_{3}$. We need to prove that there exists an $f_{1} \in G_{1}$ such that

$$
\left[\psi_{B}^{2}\left(\psi_{B}^{1}(x)\right) \wedge f_{3}\left(\psi_{B}^{2}\left(\psi_{B}^{1}(y)\right)\right)\right]_{G_{3}} \leq\left[\psi_{B}^{2}\left(\psi_{B}^{1}\left(x \wedge f_{1}(y)\right)\right)\right]_{G_{3}} .
$$

Since $\psi_{B}^{2}$ satisfies the condition $(4), \psi_{B}^{1}(x), \psi_{B}^{1}(y) \in B_{2}$ and $f_{3} \in G_{3}$ imply that there is $f_{2} \in G_{2}$ such that

$$
\left[\psi_{B}^{2}\left(\psi_{B}^{1}(x)\right) \wedge f_{3}\left(\psi_{B}^{2}\left(\psi_{B}^{1}(y)\right)\right)\right]_{G_{3}} \leq\left[\psi_{B}^{2}\left(\psi_{B}^{1}(x) \wedge f_{2}\left(\psi_{B}^{1}(y)\right)\right)\right]_{G_{3}} .
$$

By Lemma 29, there is $f_{1} \in G_{1}$ such that

$$
\left[f_{2}\left(\psi_{B}^{1}(y)\right)\right]_{G_{2}} \leq\left[\psi_{B}^{1}\left(f_{1}(y)\right)\right]_{G_{2}} .
$$

By Theorem 23, $\Delta\left(\psi_{B}^{2}\right): B_{2} / G_{2} \rightarrow B_{3} / G_{3}$ is a morphism of MV-algebras. In particular, $\Delta\left(\psi_{B}^{2}\right)$ is an isotone mapping, hence

$$
\Delta\left(\psi_{B}^{2}\right)\left(\left[f_{2}\left(\psi_{B}^{1}(y)\right)\right]_{G_{2}}\right) \leq \Delta\left(\psi_{B}^{2}\right)\left(\left[\psi_{B}^{1}\left(f_{1}(y)\right)\right]_{G_{2}}\right),
$$

that means,

This implies that

$$
\left[\psi_{B}^{2}\left(f_{2}\left(\psi_{B}^{1}(y)\right)\right)\right]_{G_{3}} \leq\left[\psi_{B}^{2}\left(\psi_{B}^{1}\left(f_{1}(y)\right)\right)\right]_{G_{3}} .
$$

$$
\left[\psi_{B}^{2}\left(\psi_{B}^{1}(x)\right) \wedge \psi_{B}^{2}\left(f_{2}\left(\psi_{B}^{1}(y)\right)\right)\right]_{G_{3}} \leq\left[\psi_{B}^{2}\left(\psi_{B}^{1}(x)\right) \wedge \psi_{B}^{2}\left(\psi_{B}^{1}\left(f_{1}(y)\right)\right)\right]_{G_{3}} .
$$

Since $\psi_{B}^{1}$ and $\psi_{B}^{2}$ are morphisms of Boolean algebras, we see that

$$
\left[\psi_{B}^{2}\left(\psi_{B}^{1}(x)\right) \wedge \psi_{B}^{2}\left(\psi_{B}^{1}\left(f_{1}(y)\right)\right)\right]_{G_{3}}=\left[\psi_{B}^{2}\left(\psi_{B}^{1}\left(x \wedge f_{1}(y)\right)\right)\right]_{G_{3}} .
$$

For an MV-algebra $M$, let us write $\nabla(M)=(B(M), G(M))$. For an MV-pair $(B, G)$ let us write $\Delta(B, G)=B / G$. 
Theorem 31. $\Delta$ is a functor.

Proof. Obviously, the identity morphisms are preserved.

Let

$$
\begin{gathered}
\psi_{B}^{1}:\left(B_{1}, G_{1}\right) \rightarrow\left(B_{2}, G_{2}\right), \\
\psi_{B}^{2}:\left(B_{2}, G_{2}\right) \rightarrow\left(B_{3}, G_{3}\right)
\end{gathered}
$$

be morphisms of MV-pairs.

By Proposition 30, $\psi_{B}^{2} \circ \psi_{B}^{1}$ is a morphism of MV-pairs. Moreover, by Theorem 23 ,

$$
\begin{aligned}
\Delta\left(\psi_{B}^{1}\right): \Delta\left(B_{1}, G_{1}\right) & \rightarrow \Delta\left(B_{2}, G_{2}\right), \\
\Delta\left(\psi_{B}^{2}\right) & : \Delta\left(B_{2}, G_{2}\right) \rightarrow \Delta\left(B_{3}, G_{3}\right) \text { and } \\
\Delta\left(\psi_{B}^{2} \circ \psi_{B}^{1}\right): \Delta\left(B_{1}, G_{1}\right) & \rightarrow \Delta\left(B_{3}, G_{3}\right)
\end{aligned}
$$

are morphisms in the category of MV-algebras.

We need to prove that $\Delta\left(\psi_{B}^{2} \circ \psi_{B}^{1}\right)=\Delta\left(\psi_{B}^{2}\right) \circ \Delta\left(\psi_{B}^{1}\right)$.

Let $[x]_{G_{1}} \in \Delta\left(B_{1}, G_{1}\right)=B_{1} / G_{1}$. Then

$$
\Delta\left(\psi_{B}^{2} \circ \psi_{B}^{1}\right)\left([x]_{G_{1}}\right)=\left[\psi_{B}^{2}\left(\psi_{B}^{1}(x)\right)\right]_{G_{3}}
$$

and

$$
\begin{array}{r}
\left(\Delta\left(\psi_{B}^{2}\right) \circ \Delta\left(\psi_{B}^{1}\right)\right)\left([x]_{G_{1}}\right)=\Delta\left(\psi_{B}^{2}\right)\left(\Delta\left(\psi_{B}^{1}\right)\left([x]_{G_{1}}\right)\right)= \\
=\Delta\left(\psi_{B}^{2}\right)\left(\left[\psi_{B}^{1}(x)\right]_{G_{2}}\right)=\left[\psi_{B}^{2}\left(\psi_{B}^{1}(x)\right)\right]_{G_{3}} .
\end{array}
$$

Theorem 32. $\nabla$ is a faithful functor.

Proof. Obviously, the identity morphisms are preserved. Let

$$
\begin{gathered}
\psi_{1}: M_{1} \rightarrow M_{2}, \\
\psi_{2}: M_{2} \rightarrow M_{3}
\end{gathered}
$$

be morphisms of MV-algebras. Then $\psi_{2} \circ \psi_{1}: M_{1} \rightarrow M_{3}$ is a morphism of MValgebras. Moreover, by Theorem 28,

$$
\begin{aligned}
\nabla\left(\psi_{1}\right): \nabla\left(M_{1}\right) & \rightarrow \nabla\left(M_{2}\right), \\
\nabla\left(\psi_{2}\right): \nabla\left(M_{2}\right) & \rightarrow \nabla\left(M_{3}\right) \text { and } \\
\nabla\left(\psi_{2} \circ \psi_{1}\right): \nabla\left(M_{1}\right) & \rightarrow \nabla\left(M_{3}\right)
\end{aligned}
$$

are morphisms of MV-pairs.

We need to prove that $\nabla\left(\psi_{2} \circ \psi_{1}\right)=\nabla\left(\psi_{2}\right) \circ \nabla\left(\psi_{1}\right)$. Essentially, this follows from the fact that $B$ is a functor from the category of bounded distributive lattices to the category of Boolean algebras. This is a well-known fact. We include the simple proof just for the convenience of the reader.

Let $x \in B\left(M_{1}\right)$, let $\left(x_{i}\right)_{i=1}^{2 n}$ be its $M_{1}$-chain representation:

$$
x=\left(x_{2} \backslash x_{1}\right) \dot{\vee} \cdots \vee\left(x_{2 n} \backslash x_{2 n-1}\right) .
$$

Then

$$
\nabla\left(\psi_{2} \circ \psi_{1}\right)=\left(\psi_{2}\left(\psi_{1}\left(x_{2}\right)\right) \backslash \psi_{2}\left(\psi_{1}\left(x_{1}\right)\right)\right) \dot{\vee} \ldots \dot{\vee}\left(\psi_{2}\left(\psi_{1}\left(x_{2 n}\right)\right) \backslash \psi_{2}\left(\psi_{1}\left(x_{2 n-1}\right)\right)\right)
$$


and

$$
\begin{aligned}
& \left(\nabla\left(\psi_{2}\right) \circ \nabla\left(\psi_{1}\right)\right)(x)=\nabla\left(\psi_{2}\right)\left(\nabla\left(\psi_{1}\right)(x)\right)= \\
= & \nabla\left(\psi_{2}\right)\left(\nabla\left(\psi_{1}\right)\left(\left(x_{2} \backslash x_{1}\right) \dot{V} \ldots \dot{\vee}\left(x_{2 n} \backslash x_{2 n-1}\right)\right)\right)= \\
= & \nabla\left(\psi_{2}\right)\left(\left(\psi_{1}\left(x_{2}\right) \backslash \psi_{1}\left(x_{1}\right)\right) \dot{\vee} \ldots \dot{\vee}\left(\psi_{1}\left(x_{2 n}\right) \backslash \psi_{1}\left(x_{2 n-1}\right)\right)\right)= \\
= & \left(\psi_{2}\left(\psi_{1}\left(x_{2}\right)\right) \backslash \psi_{2}\left(\psi_{1}\left(x_{1}\right)\right)\right) \dot{\vee} \ldots \dot{\vee}\left(\psi_{2}\left(\psi_{1}\left(x_{2 n}\right)\right) \backslash \psi_{2}\left(\psi_{1}\left(x_{2 n-1}\right)\right)\right)
\end{aligned}
$$

To prove that $\nabla$ is faithful, let $M_{1}, M_{2}$ be MV-algebras. We need to prove that the mapping $\nabla_{M_{1}, M_{2}}: \operatorname{Hom}\left(M_{1}, M_{2}\right) \rightarrow \operatorname{Hom}\left(\nabla\left(M_{1}\right), \nabla\left(M_{2}\right)\right)$ is injective. Let $\psi_{1}, \psi_{2} \in \operatorname{Hom}\left(M_{1}, M_{2}\right)$ be such that $\nabla\left(\psi_{1}\right)=\nabla\left(\psi_{2}\right)$. Since, for every $\psi \in$ $\operatorname{Hom}\left(M_{1}, M_{2}\right), \nabla(\psi)$ is an extension of $\psi$ from $M_{1}$ to $B\left(M_{1}\right), \psi_{1}=\psi_{2}$.

Theorem 33. For every $M V$-algebra $M$, write $\eta_{M}: M \rightarrow B(M) / G(M), \eta_{M}=$ $\beta_{M}^{-1}$. Then

$$
\eta: 1_{M V} \approx \Delta \nabla
$$

is a natural equivalence, where $1_{M V}$ is the identity functor on the category of $M V$ algebras.

Proof. $\eta_{M}$ is an isomorphism of MV-algebras. By Lemma 24 and Corollary 27, the diagram

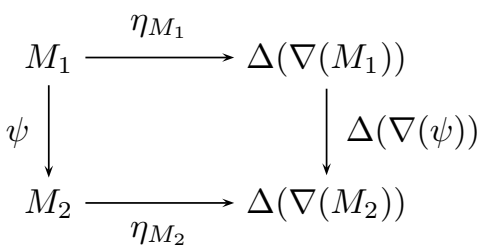

commutes.

\section{REFERENCES}

[1] M.K. Bennett and D.J. Foulis. Phi-symmetric effect algebras. Foundations of Physics, 25:1699-9722, 1995.

[2] C.C. Chang. Algebraic analysis of many-valued logics. Trans. Amer. Math. Soc., 88:467-490, 1959.

[3] G. Chevalier and S.Pulmannová. Some ideal lattices in partial abelian monoids. Order, 17:7592, 2000.

[4] F. Chovanec and F. Kôpka. Boolean D-posets. Tatra Mt. Math. Publ, 10:183-197, 1997.

[5] R. Cignoli, E. Dubuc, and D. Mundici. The MV-algebraic invariant for Boolean algebras with finite-orbit automorphism. Tatra Mt. Math. Publ, 27:23-44, 2003.

[6] B. K. Dauletbaev. Determining an atomic Boolean algebra from the action of the automorphism group. Siberian Mathematical Journal, 34(6):1041-1043, 1993.

[7] A. Dvurečenskij and S. Pulmannová. New Trends in Quantum Structures. Kluwer, Dordrecht and Ister Science, Bratislava, 2000.

[8] D.J. Foulis and M.K. Bennett. Effect algebras and unsharp quantum logics. Found. Phys., 24:1325-1346, 1994.

[9] R. Giuntini and H. Greuling. Toward a formal language for unsharp properties. Found. Phys., 19:931-945, 1989.

[10] G. Grätzer. General Lattice Theory. Birkhäuser, second edition, 1998.

[11] S. Gudder and S. Pulmannová. Quotients of partial abelian monoids. Algebra univers., 38:395-421, 1998.

[12] J. Hashimoto. Ideal theory for lattices. Math. Japon., 2:149-186, 1952.

[13] G. Jenča. Boolean algebras R-generated by MV-effect algebras. Fuzzy sets and systems, $145: 279-285,2004$ 
[14] G. Jenča. A representation theorem for MV-algebras. Soft Computing, 11:557-564, 2007.

[15] F. Kôpka. D-posets of fuzzy sets. Tatra Mt. Math. Publ., 1:83-87, 1992.

[16] F. Kôpka and F. Chovanec. D-posets. Math. Slovaca, 44:21-34, 1994.

[17] S. Koppelberg. Handbook of Boolean Algebras. North-Holland, 1989.

[18] H.M. MacNeille. Extension of a distributive lattice to a Boolean ring. Bull. Amer. Math. Soc., 45:452-455, 1939.

[19] J. Donald Monk. Automorphism groups. In J. Donald Monk and Robert Bonnet, editors, Handbook of Boolean algebras, volume 2, chapter 14, pages 517-545. North-Holland, 1989.

[20] A. S. Morozov. Groups of recursive automorphisms of constructive boolean algebras. Algebra and Logic, 22(2):95-112, 1983.

[21] A. S. Morozov and B. Kasymkhanuly. Boolean algebras with finite families of computable automorphisms. Siberian Mathematical Journal, 45(1):141-145, 2004.

[22] D. Mundici. Interpretation of $\mathrm{AF} C^{*}$-algebras in Lukasziewicz sentential calculus. J. Functional Analysis, 65:15-53, 1986.

[23] P. Niederkorn. Natural dualities for varieties of MV-algebras. J. Math. Anal. Appl., 255:5873, 2001.

[24] A. Di Nola and A. Lettieri. One chain generated varieties of MV-algebras. Journal of Algebra, 225:667-697, 2000.

[25] S. Pulmannová. Congruences in partial abelian semigroups. Algebra univers., 37:119-140, 1997.

[26] S. Pulmannová and E. Vinceková. Ideals in MV-pairs. Soft Computing, 12:1199-1204, 2008.

[27] Matayahu Rubin. On reconstruction of the boolean algebras from their automorphism groups. In J. Donald Monk and Robert Bonnet, editors, Handbook of Boolean algebras, volume 2, chapter 14, pages 547-605. North-Holland, 1989.

[28] Thomas Vetterlein. Boolean algebras with an automorphism group: a framework for łukasiewicz logic. J. Mult.-Val. Log. Soft Comput., 14:51-67, 2008.

(A. Di Nola) Department of Mathematics and Information Sciences, Università degli Studi di Salerno, Via Ponte Don Melillo, 84084 Fisciano (Salerno), Italy

E-mail address: adinola@unisa.it

(M. Holčapek) Institute for Research and Applications of Fuzzy Modeling, University of Ostrava, 30. dubna 22, 70133 Ostrava 1, Czech Republic

E-mail address: michal.holcapek@osu.cz

(G. Jenča) Department of Mathematics and Descriptive Geometry, Faculty of Civil Engineering, Radlinského 11, Bratislava 813 68, Slovak Republio

E-mail address: gejza.jenca@stuba.sk 